\title{
A Continuous Timed Petri Net Model and Control for a
}

\section{Gas Turbine}

\author{
Alexander Núñez, Eloy Edmundo Rodríguez and Ottmar Uriza \\ LaNITEF National Laboratory of Cooling Research and Technologies, Engineering and Industrial Development Center, Pie de la \\ cuesta 702, Col. Desarrollo San Pablo, Querétaro, Qro, México
}

\begin{abstract}
This work developed the modeling and supervisory control for gas turbine. A CTPN (continuous timed Petri Net) model of a gas turbine, using a first linear order approximation for every state of the Brayton cycle is obtained. The Brayton cycle rules the functioning of a gas turbine, and it is composed by four states: compression, combustion, expansion and cooling. The principle of the gas turbine is developed by the Brayton cycle, a thermodynamic process which intervenes in the gas turbine components. The steady-state behavior of the gas turbine has been widely investigated in engineering area. Moreover, the dynamic behavior has been studied using non-linear models of its components, leading to complicated mathematical representations. The methodology of the current work begins with a simplification of the dynamical relations in every state (excepting the cooling phase) of the Brayton cycle. Temperature and pressure are modeled as first order linear systems, therefore, every system is translated into a CTPN. Furthermore, to guarantee a safety operation, an SC (supervisory controller) is designed to ensure the combustion chamber temperature is lower than $1,000{ }^{\circ} \mathrm{C}$. Although the model presented is extremely simplified, it will be used as a starting point to develop more complex models.
\end{abstract}

Key words: SCT (Supervisory control theory), PNs (Petri Nets), gas turbine, Brayton cycle.

\section{Introduction}

The gas turbine thermodynamic and mechanic nature are considered dynamic but the control algorithms are referred to specific punctual measurements, making the control algorithm work as a supervisor instead of a dynamic regulator. In this scenario, the need of modeling the gas turbine system as a DES (discrete event system) has become greater in the latest years. The design of SCs (supervisory controllers) with PNs (Petri Nets) using the IBCD (invariant based control design) method $[1,2]$ has been previously considered for the avoidance of forbidden states [3] and for constraining the system behavior using linear in-equalities induced by BCs (behavioral constraints) [4]. The application of SCT (supervisory control theory) has been studied in Ref. [5] for manufacturing uses. Plenty of works are

Corresponding author: Alexander Núñez, Ph.D. candidate, research fields: dynamic systems, Petri Nets, control theory, mathematical modeling, thermodynamic systems. dedicated to analyzing the behavior of the so-called hybrid systems, using as main approach continuous and fluid PNs. In Ref. [6], a non-deterministic model of a temperature control system is developed. In this work, the assumption that the gas turbine is in fact a deterministic system is strongly considered, in order to propose simplified first order models. Gas turbines are used in transport systems as aircraft and in power generating systems. The principle of the gas turbine is developed by the Brayton cycle, a thermodynamic process which intervenes in the gas turbine components. The steady-state behavior of the gas turbine has been widely investigated in engineering area. Moreover, the dynamic behavior has been studied using non-linear models of its components, leading to complicated mathematical representations.

However, it is impossible to ignore the dynamic behavior of the system during the Brayton cycle, so a standard modeling of the gas turbine as discrete state system is not enough to capture its behavior. The full analysis of the CTPN (continuous timed Petri Net) 
model is developed in Ref. [7]. In Section 2, the basic concepts of gas turbines, continuous PNs and SC are introduced. The mathematical reduction and the CTPN model of the gas turbine dynamics is presented in Section 3. In Section 4 an SC is designed using the IBCD to ensure a combustion chamber temperature bounded by $1,000{ }^{\circ} \mathrm{C}$ and a simulation is obtained in Mat-lab. Finally, the conclusions and further work are presented in Section 5 .

\section{Fundamentals}

Gas turbine, Brayton cycle and SCT are briefly introduced in this section.

\subsection{Gas Turbine}

A generic gas turbine is composed by three elements, each one is associated to a state of the Brayton cycle. The elements are shown in Fig. 1. For further reading, review Refs. [8, 9].

\subsection{Brayton Cycle}

The Brayton cycle is used to define the behavior of a gas turbine. It is composed by four states:

(1) Ompression. Ideally it is an adiabatic process (no heat transfer with the environment). However, the temperature does increase, as has been shown in several experiments;

(2) Heat addition (combustion). An isobaric process (no change on the fluid volume);

(3) Expansion. Again, an ideally adiabatic process;

(4) Heat rejection. An isobaric process.

For simplicity of the measurements, entropy is not included in the present analysis. The graphical representation of Brayton Cycle is presented in Fig. 2.

This description is remarkable that each state occurs in a component of the gas turbine.

\section{$2.3 C T P N$}

The following subsection has been taken directly from Ref. [10]. The reader is refereed to Refs. [11, 12] for related subjects in CTPNs.

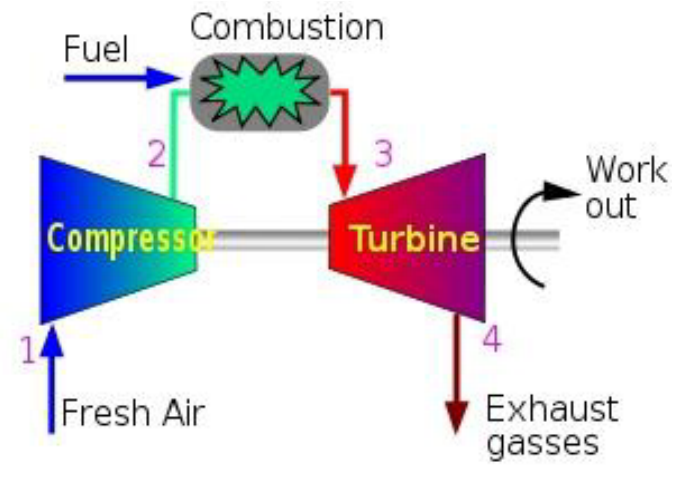

Fig. 1 Gas turbine.

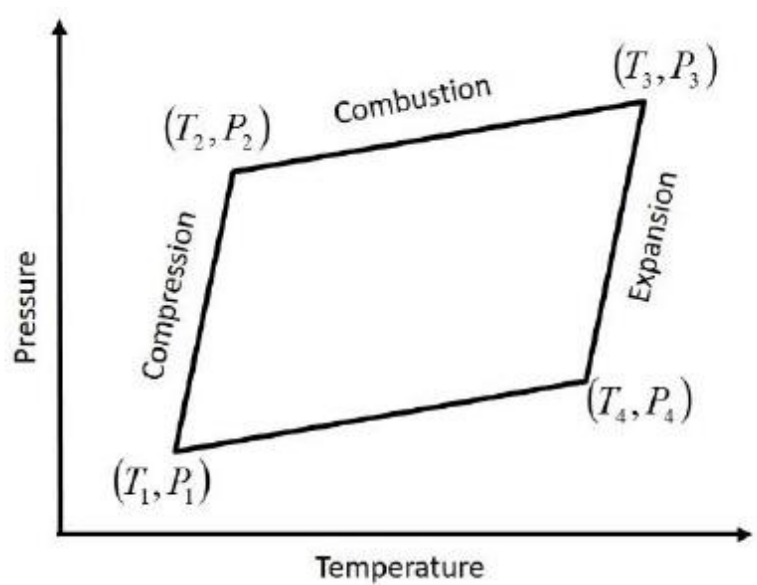

Fig. 2 Brayton cycle.

A CTPN is a 3-tuple ContPN $=\left(N, \lambda, m_{0}\right)$, where $(N$, $\left.m_{0}\right)$ is a continuous PN and $\lambda: T \rightarrow\left\{R^{+}\right\}$is a function associating a firing rate with each transition.

The state equation of a ContPN is shown in Eq. (1)

$$
\dot{m(\tau)}=C * f(\tau)
$$

where, $\tau$ is the time variable, $C$ is the net incidence matrix, $C=$ Post - Pre.

A ContPN is called infinite server semantic if the flow of a transition $t_{i}$ is

$$
f_{i}=\lambda_{i} * \min _{p \in \operatorname{pre}(t i)}\left\{m(p) \mid \operatorname{Pr} e\left[p, t_{i}\right]\right\}
$$

Eq. (1) can be expressed as a piecewise linear system given by

$$
\dot{m}=C \Lambda \Pi(m) m
$$

The firing rate matrix is denoted by $\Lambda=\operatorname{diag}$ $\left(\lambda_{1}, \ldots, \lambda_{\mid T}\right)$. A configuration of a ContPN at $m$ is a set of $(p, t)$ arcs describing the effective flow of all transitions:

$$
\Pi(\mathrm{m})[\mathrm{i}, \mathrm{j}]=\left\{\begin{array}{cc}
\frac{1}{\text { Pre }[i, j]} & \text { if } p_{i} \text { is constrainig } t_{j} \\
0 & \text { otherwise }
\end{array}\right.
$$




\section{$2.4 S C T$}

The automata version of SCT is developed in Ref. [13]. In this subsection, the fundamentals SCT for discrete event system modeled as Petri Net are introduced.

Definition 1 (control pattern). Let $\mathrm{N}$ be a PN and $T$ be its set of transitions.

The control pattern is defined as the set of transitions enabled in a marking $M$ of $(N, M)$.

Definition 2 (transition sequence). Let $(N, M)$ be a PN system and $T$ be its set of transitions.

$\sigma=t_{1} t_{2} \cdots \cdots t_{\mathrm{n}}$ is a transition sequence of transitions such that

- $M_{j-1} \stackrel{t_{i}}{\rightarrow} M_{j}$

- $t_{i}$ is enabled in $M_{i-1}$

with $t_{i} \in T, \forall i=1,2, \cdots \cdots, n$.

Definition 3 (Petri Net supervisor). Consider a constraint $L * M \leq b$ for the marking vector of a PN system $(N, M)$ with incidence matrix $D$ and $L$ a matrix corresponding the set of inequalities in the constraint. $S: M \rightarrow$ is a supervisor for $P N$ system $(N, M)$. Let $C$ be a PN with marking $M_{\mathrm{c}}$ and set of transition $T$. $C$ is the implementation of $S$ as a PN such that

- Marking vector $M c=\mathrm{b}-L * M_{0}$;

- Incidence matrix $D c=-L * D$;

- is the control pattern for $\left(C, M_{\mathrm{c}}\right)$.

Definition 4 (admissible marking). Let marking $M^{0}$ be reachable from $M_{0} . M^{0}$ is an admissible marking for the constraint $L * M \leq b$ if

- $L * M^{0} \leq b$

- For all reachable markings $M_{r}$ from $M_{0}$ trough the occurrence of uncontrollable transitions in $(N, M)$ $L * M_{r} \leq b$.

Definition 5 (admissible constraint). Let $(N, M)$ be a PN system with initial marking $M_{0}$. An admissible constraint satisfies

- $L * M_{0} \leq b$

- All reachable markings from $\mathrm{M}_{0}$ are admissible markings.
Corollary 6. Given a plant with uncontrollable transitions represented by its incidence matrix $D_{p}$, it is partitioned in the following form

$$
D_{p}=\left[D D_{u c}\right]
$$

with $D$ representing the columns of controllable transitions and $D_{u c}$ uncontrollable transitions.

Let $L * M_{\mathrm{p}} \leq b$ be a marking constraint. If Eq. (5) holds then constraint is admissible.

$$
L * D_{u c} \leq 0
$$

\section{CTPN Model}

In this section the model of the Brayton cycle as in hybrid system is developed. A high-level discrete event system is constructed using the four states of the Brayton cycle, explained in Section 2.2. In each state, the dynamic equations of temperature and pressure are obtained, based on the thermodynamical relationships introduced in Ref. [14].

\subsection{High-Level Discrete State Model}

Brayton cycle is composed by 4 states:

(1) Compression;

(2) Combustion;

(3) Expansion;

(4) Cooling.

In addtion to these states, an initial state representing no operation of the gas turbine is included in the model. The corresponding finite state machine of the Brayton cycle is presented in Fig. 3.

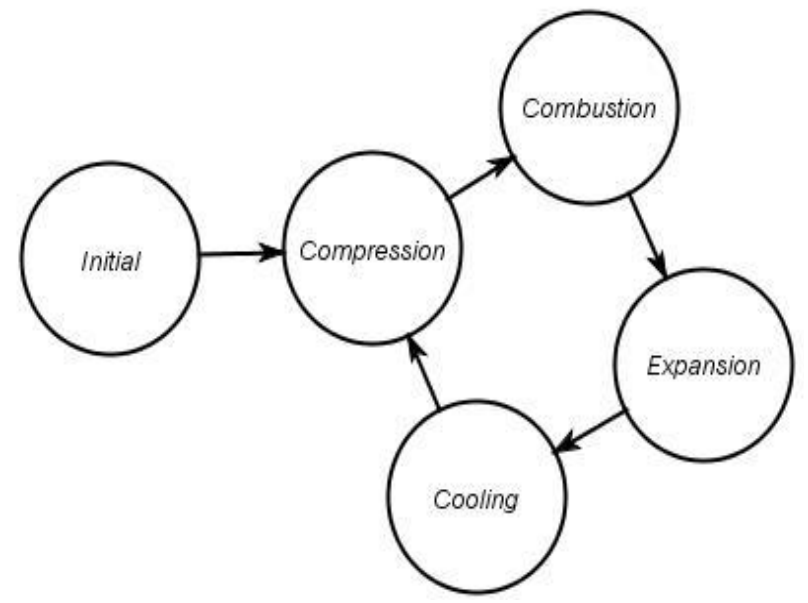

Fig. 3 Brayton cycle modeled as a Finite State Machine. 


\subsection{Mathematical Representation of Temperature and}

\section{Pressure}

In each state of the system, the temperature and pressure evolve according to dynamical non-linear equations. For this work, the relationships have been reduced into first order linear dynamical equations in the form of Eq. (6).

$$
\dot{m}=-\alpha m+\kappa u
$$

where, $\alpha>0$ and $\kappa>0$.

With $u$ an input variable which increases the value of $f$, the coefficients $\alpha$ and $\kappa$ are determined based on experimental results of the temperature and pressure behavior in each state.

\subsubsection{Compression Dynamic Equations}

In the state of compression, the input variable is the angular speed $\omega$ of the compressor and it increases the value of the pressure in the output of the compressor $P_{2}$, giving result as Eq. (7).

$$
\dot{P}_{2}-\alpha P_{2} P_{2}+\kappa P_{2} \omega
$$

With initial condition of the atmospheric pressure $P_{1}$, the thermodynamic relationship between pressure and temperature into the compressor is developed in Ref. [14] and is presented in Eqs. (8) and (9).

$$
\begin{gathered}
K_{c}(t)=\frac{P_{2}}{P_{1}} \\
T_{2}=K_{c} T_{1}
\end{gathered}
$$

$T_{2}$ is the temperature in the output of compressor and $T_{1}$ the environment temperature. From Eqs. (8) and (9)

$$
T_{2}=\frac{P_{2}}{P_{1}} T_{1}
$$

Considering $P_{1}$ and $T_{1}$ constants (this assumption is valid because of the slow rate change of environmental conditions) and differentiating Eq. (10)

$$
\begin{gathered}
\dot{T}_{2}=\frac{\dot{P_{2}}}{P_{1}} T_{1} \\
\dot{T_{2}}=\frac{T_{1}}{P_{1}}\left(-\alpha P 2 P_{2}+\kappa P 2 \omega\right) \\
\dot{T}_{2}=-\alpha P 2 T_{2}+\frac{T_{1}}{P_{1}} \kappa P 2 \omega
\end{gathered}
$$

\subsubsection{Combustion Dynamic Equations}

Temperature in the output of combustion chamber $T_{3}$ evolves with the applied heat $Q$ generated by the ignition. $Q$ is proportional to the amount of air mass in the combustion chamber, which is proportional to the open percentage of angle of the stator position $\theta_{\text {Air. }}$ Eq. (14) represents the evolution of $T_{3}$ with a control input $\theta_{\text {Air. }}$

$$
\dot{T}_{3}=-\alpha T 3\left(T_{3}-T_{1}\right)+\kappa T 3 \theta_{\text {Air }}
$$

Pressure in the output of combustion chamber $P_{3}$ evolves according to the heat transference, such as $T_{3}$, leading to Eq. (15)

$$
\dot{P}_{3}=-\alpha P 3\left(P_{3}-P_{2}\right)+\kappa P 3 \theta_{\text {Air }}
$$

\subsubsection{Expansion Dynamic Equations}

Similarly to compression, pressure $P_{4}$ in the output of the turbine is represented as a first order linear system with a control input $\omega$, as seen in Eq. (16). Making an analysis of the temperature exchange in this process similar to compression process, Eq. (17) represents the temperature behavior in the output of the turbine, considering $T_{3}$ and $P_{3}$ as constants during the expansion process.

$$
\begin{gathered}
\dot{P}_{4}-\alpha P 4 P_{4}+\kappa P 4 \omega \\
\dot{T}_{4}=-\alpha P 4 T_{2}+\frac{T_{3}}{P_{3}} \kappa P 3 \omega
\end{gathered}
$$

Brayton cycle for gas turbine is not a closed process, therefore the air in the output of the turbine is not reused as input for the compressor. Thus, the cooling dynamics is not necessary to analyze the system behavior.

Using the equations developed previously, CTPN models are described as it follows.

\subsection{Compressor Petri Net Model}

Using Eqs. (13) and (17) the Petri Net model shown in Fig. 4 is developed.

\subsection{Combustion Chamber Petri Net Model}

Using Eqs. (14) and (15) the Petri Net model shown in Fig. 5 is developed. 


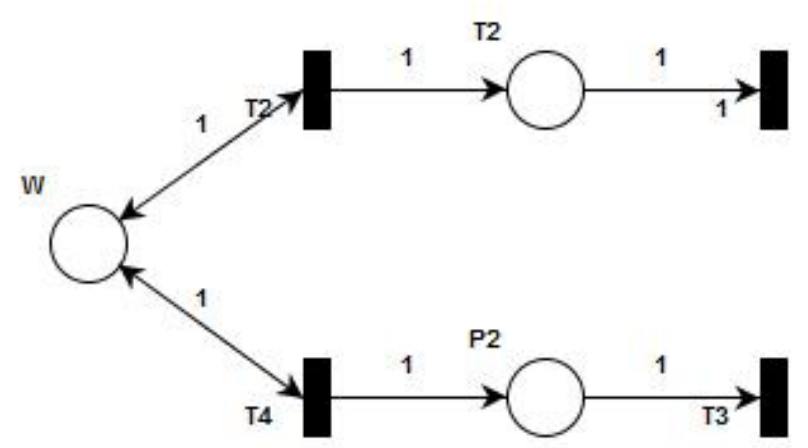

Fig. 4 Compressor Petri Net model.

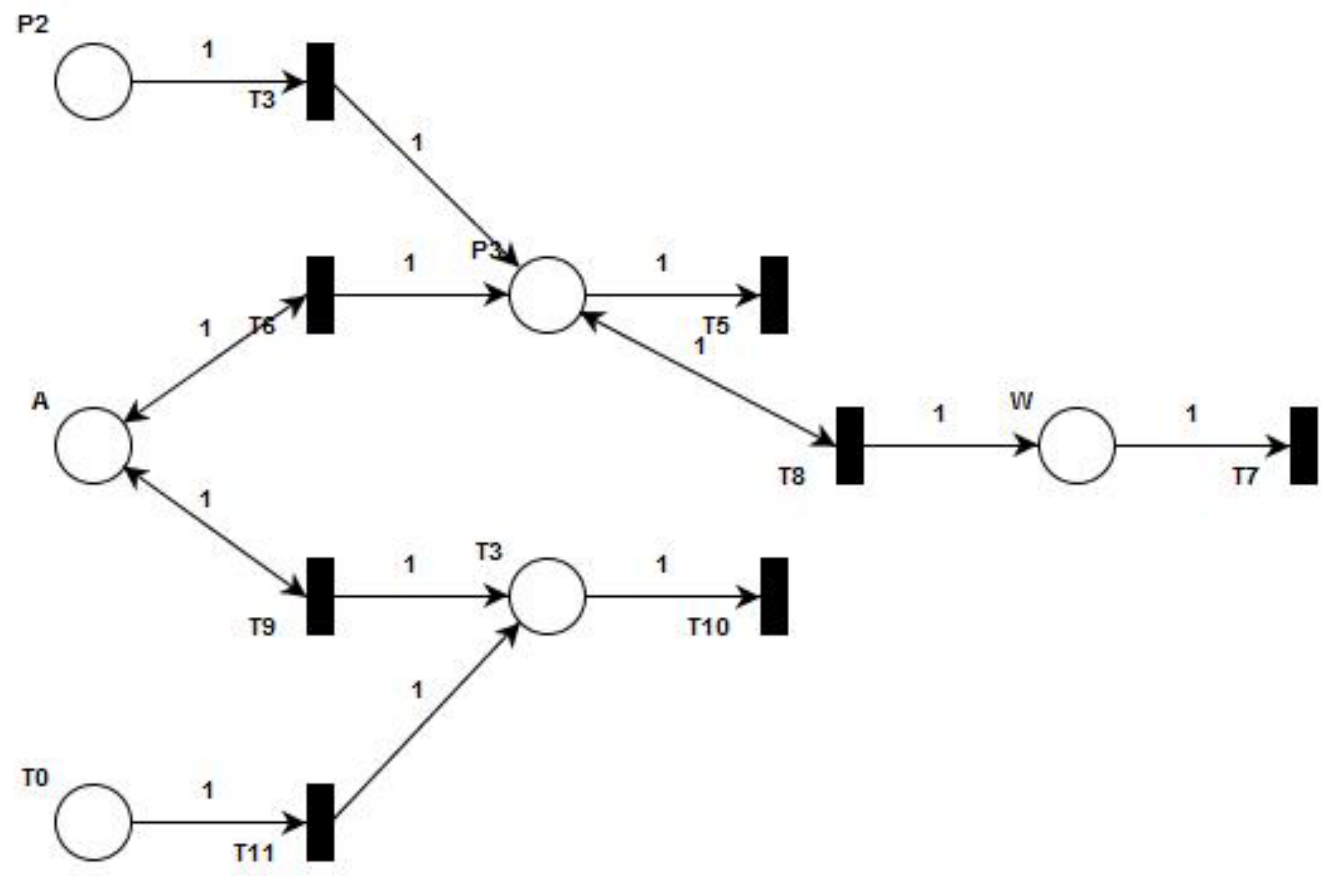

Fig. 5 Combustion chamber Petri Net model.

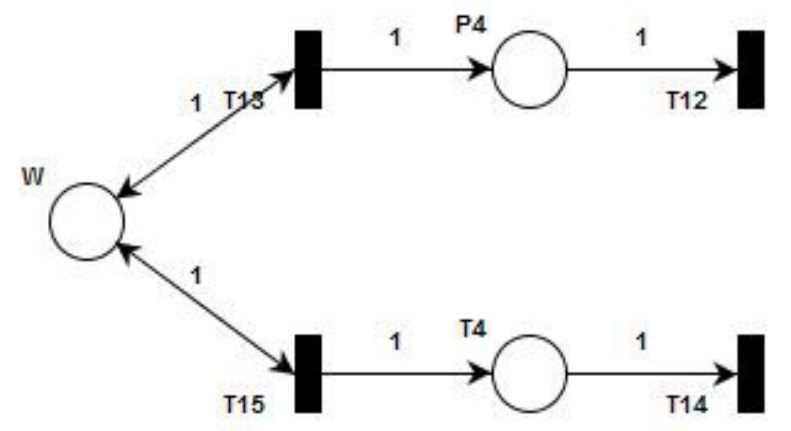

Fig. 6 Turbine Petri Net model.

\subsection{Turbine Petri Net Model}

Using Eqs. (16) and (17) the Petri Net model shown in Fig. 6 is developed.

Merging the models shown above, a complete gas turbine Petri Net model is obtained with the following incidence matrix $C$ and firing rate matrix $\Lambda$, using the place index as shown in Table 1.

Table 1 Turbine Petri Net model.

\begin{tabular}{|l|l|}
\hline$M_{1}$ & $P_{2}$ \\
\hline$M_{2}$ & $T_{2}$ \\
\hline $\mathrm{M}_{3}$ & $\Omega$ \\
\hline$M_{4}$ & $P_{3}$ \\
\hline$M_{5}$ & $\theta_{\text {Air }}$ \\
\hline$M_{6}$ & $T_{3}$ \\
\hline$M_{7}$ & $T_{0}$ \\
\hline$M_{8}$ & $P_{4}$ \\
\hline$M_{9}$ & $T_{4}$ \\
\hline
\end{tabular}




\subsection{Open Loop Simulation Results}

Using SIMHPN matlab toolkit [15], the dynamic models of each state in the Brayton cycle are simulated using the values presented in Eq. (19).

The results are shown in Figs. 7-13 and any constraint is enforced to the system. In the beginning of the process, a gear is used to start the compression stage.

Once in steady state, the gear is off and the system is sustained by the air flux, which is submitted to a Brayton cycle as described in Fig. 14.

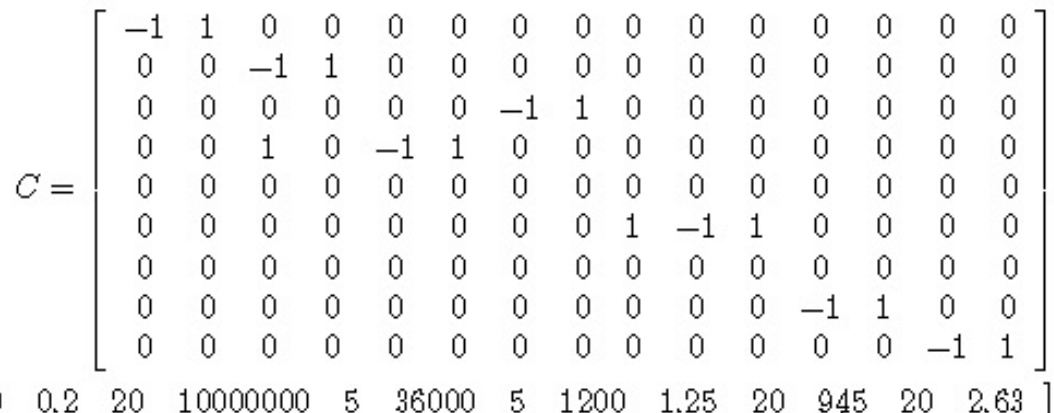

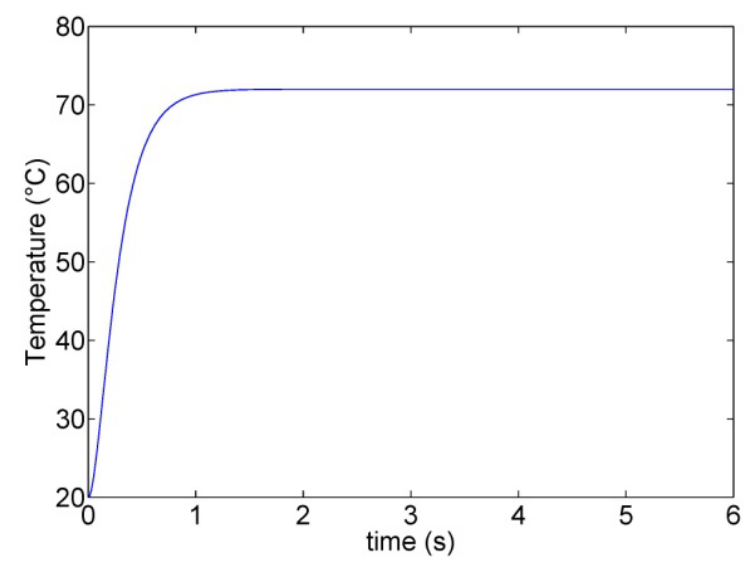

Fig. $7 T_{2}$ (temperature in the output of compressor).

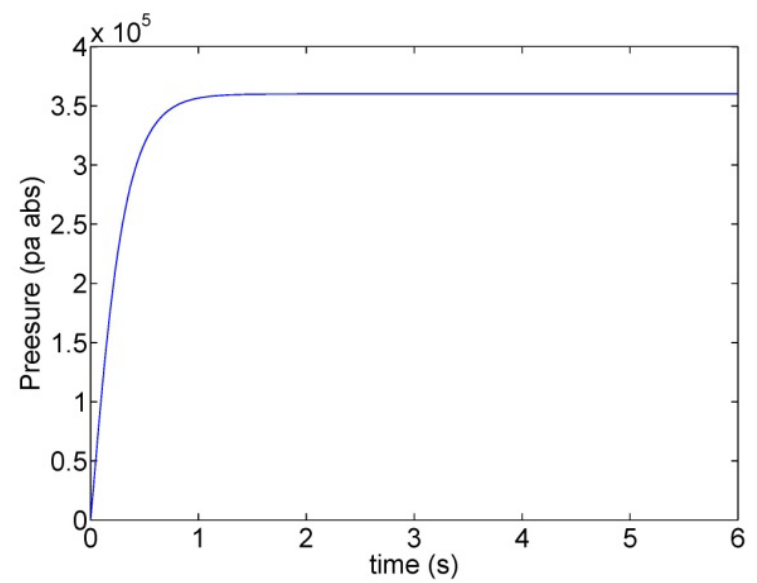

Fig. $8 P_{2}$ (pressure in the output of compressor).

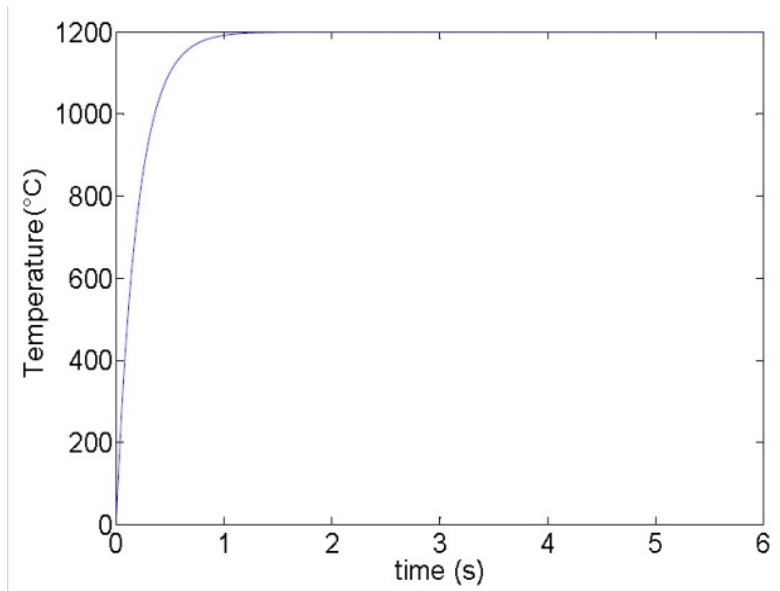

Fig. $9 T_{3}$ (temperature in the output of combustor).

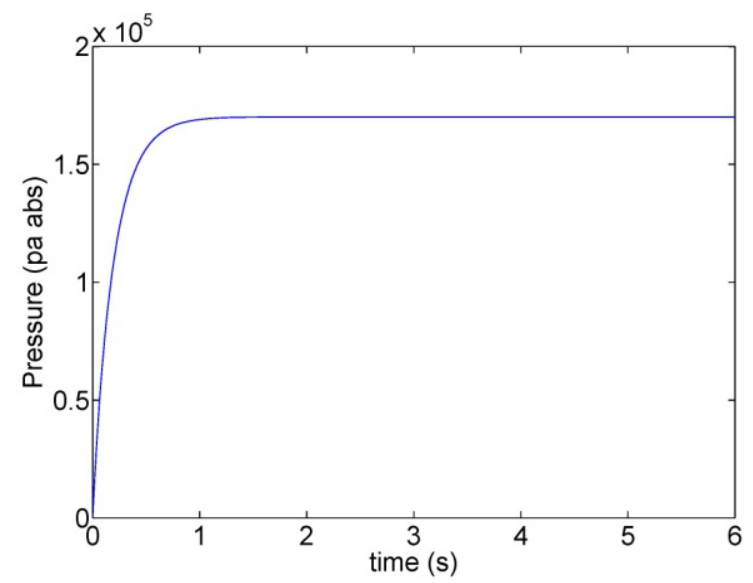

Fig. $10 P_{4}$ (pressure in the output of turbine). 


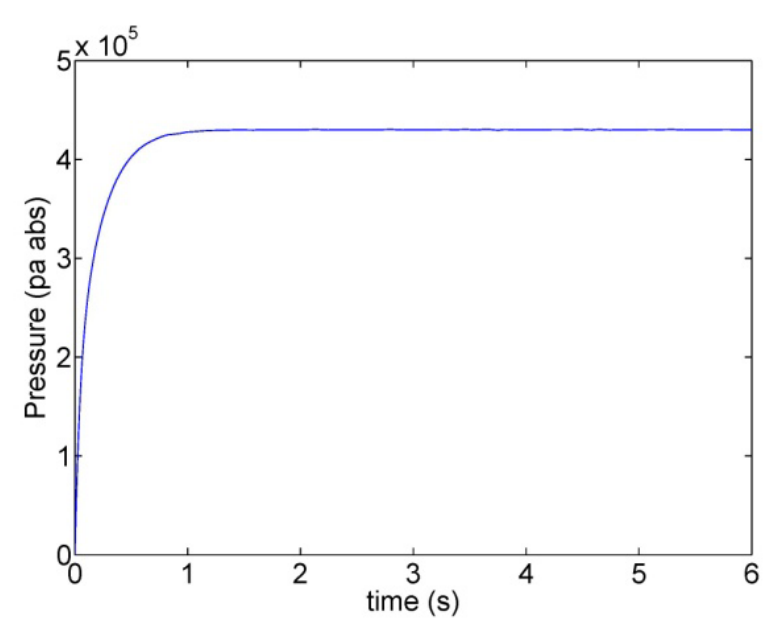

Fig. $11 P_{3}$ (pressure in the output of compressor).

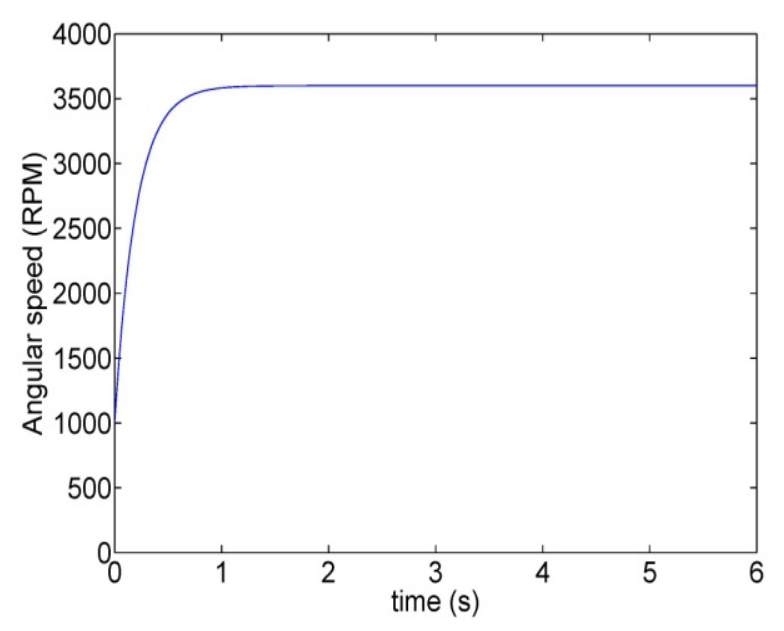

Fig. $12 \omega$ (angular speed of the rotor).

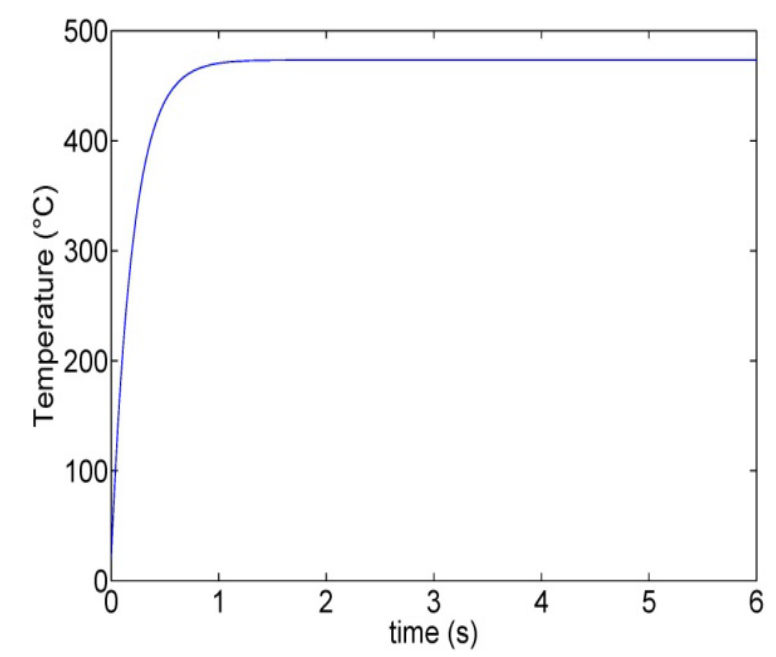

Fig. $13 T_{4}$ (temperature in the output of turbine).

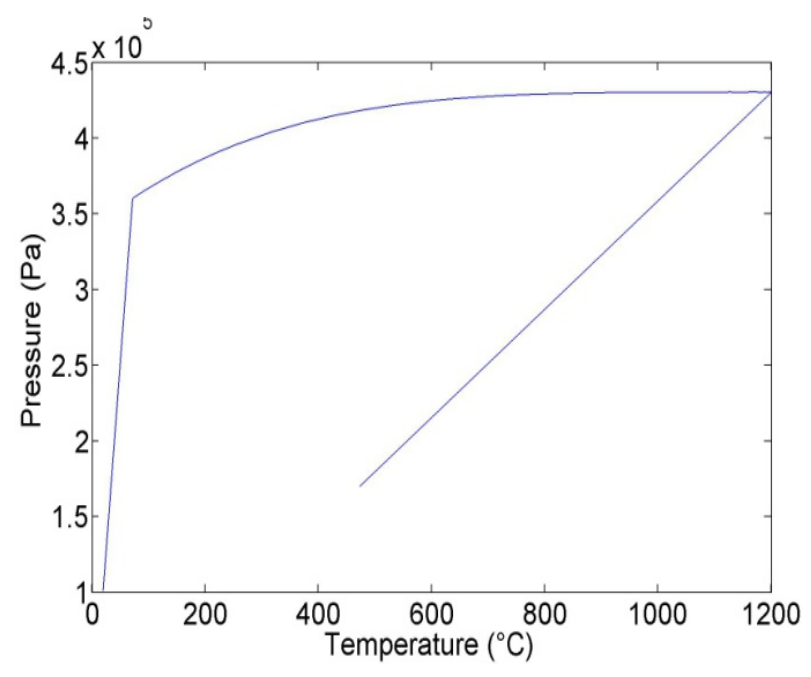

Fig. 14 Brayton cycle simulated using CTPN models.

\section{SC Design}

There are plenty of safety specifications that can be enforced into the gas turbine dynamics, such as regulate the temperature and pressure of every state, or sustain a constant angular speed in the rotor.

For the propose of this work, the safety specifications enforce the system to regulate the combustion chamber temperature and keep it lower than $1,000{ }^{\circ} \mathrm{C}$. The temperature is selected according to the open loop behavior of the gas turbine, reaching a combustion chamber temperature of $1,300{ }^{\circ} \mathrm{C}$, the bound may be changed and the design process would not change significantly.

The constraint used in this case involves the combustion chamber temperature $T_{3}$, which according to Table 1 is represented by place 6 . Hence, the algebraic constraint is presented in Eq. (20).

$$
M_{6} \geq 1,000
$$

Therefore, the matrix $L$ and the value $b$ are obtained to apply IBCD. The initial marking of place 6 is environmental temperature, $20^{\circ} \mathrm{C}$.

$$
\begin{aligned}
L=\left[\begin{array}{ccccccccc}
0 & 0 & 0 & 0 & 0 & 1 & 0 & 0 & 0
\end{array}\right] \\
b=1,000 \\
D_{c}=L * C
\end{aligned}
$$

Using Eqs. (23) and (25), the incidence matrix $D_{c}$ and initial marking $M_{c 0}$ of the monitor place are calculated, considering $C$ as the system incidence 
matrix and $M_{0}$ as system initial marking.

\subsection{Admissibility Analysis}

The SC designed in previous section is only physically implementable if the constraint is admissible. Corollary 6 provides a useful proof in order to determine if the constraint is admissible. First, it is necessary to define the controllable and uncontrollable transitions in the gas turbine. The only controllable transition is associated to angle of the gas valve. This transition has been numbered with the index $T_{3}$. According to the incidence matrix, its corresponding column is column number 9 . Then, the application of Eq. (5) leads to Eq. (27).

Since all the entries of this matrix are less than zero, the constraint is admissible.

In Fig. 15 a simulation using Matlab toolbox is shown.

$$
\begin{aligned}
& D_{c}=\left[\begin{array}{llllllllllllll}
0 & 0 & 0 & 0 & 0 & 0 & 0 & 0 & 0 & 1-1 & -1 & 0 & 0 & 0
\end{array}\right] \\
& M_{c o}=b-L * M_{o} \\
& M_{c o}=1000-20=980
\end{aligned}
$$

\section{Conclusions and Further Work}

The design of SC provides a technique to enforce system behavior into a gas turbine. The representation as a CTPN is commonly used for discrete event systems in order to apply supervisory control to constraint the system behavior. A comparison between the open-loop system and the supervised one is established using SIMHPN matlab toolkit [15].
Defining marking constraints or BCs, the system can be submitted to a specification behavior. The constraints can directly forbid certain place to reach a value, such as temperature must be always below $1,000{ }^{\circ} \mathrm{C}$ or disable some action in order to prevent a bad performance, such as cut the air or the fuel supply if the angular speed is increasing. The formal techniques of supervisory control are widely developed and the approach presented in this work allows the synthesis of SCs for a gas turbine. Using the approach as a simplified model is significant in order to add non-linear behavior. Another significant application of PNs is developing models to estimate failures and diagnosis on running on-line systems, as is shown in Ref. [16]. A future research including both PNs approaches improves the reliability of a gas turbine and its control algorithms.

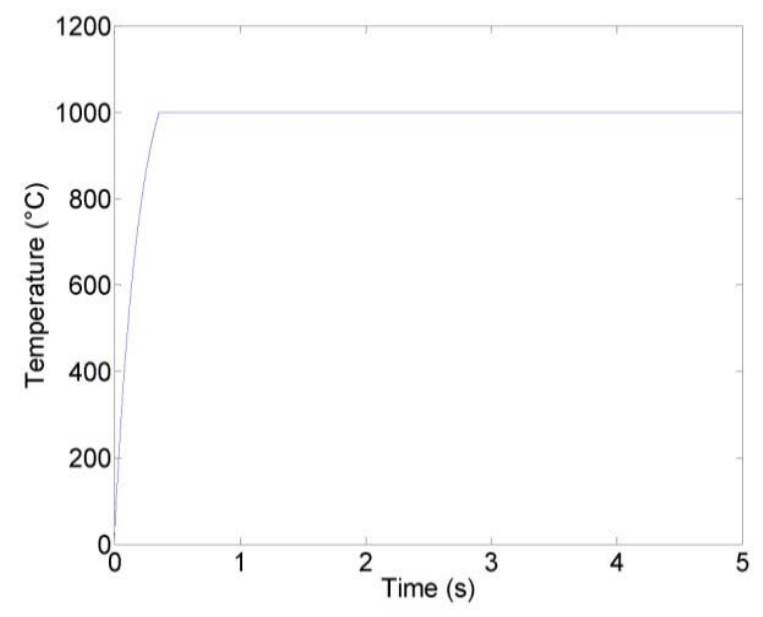

Fig. 15 Temperature in combustion chamber.

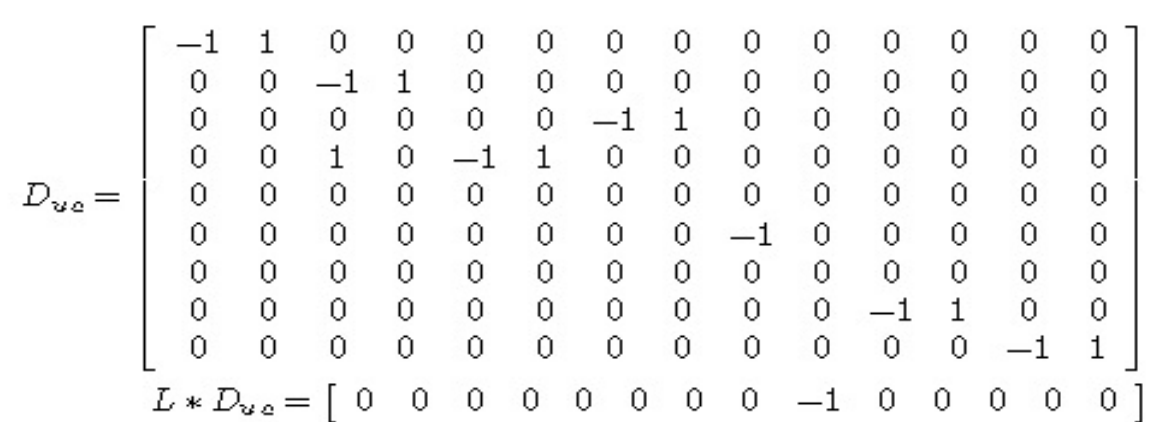




\section{Acknowledgements}

The present work has been funded by the National Laboratory in Cooling Technologies and Research (LANITEF), consolidated in project 280500 of CONACYT.

\section{References}

[1] Moody, J. O., and Antsaklis, P. J. 1998. Supervisory Control of Discrete Event System Using Petri Nets. Dordrecht: Kluwer Academic Publishers.

[2] Iordache, M. V., and Antsaklis, P. J. 2006. Supervisory Control of Concurrent Systems: A Petri Net Structural Aproach. Cambridge: Birkhäuser.

[3] Giua, A., DiCesare, F., and Silva, M. 1992. "Generalized Mutual Exclusion Contraints on Nets with Uncontrollable Transitions." IEEE International Conference on Systems, Man, and Cybernetics 2: 974-9.

[4] Yamalidou, E., and Kantor, J. 1991. "Modeling and Optimal Control of Discrete-Event Chemical Processes Using Petri Nets." Computers and Chemical Engineering 15 (7): 503-19.

[5] Núñez, A., and Sánchez, A. 2015. "Supervisory Control Based on Behavioral Constraints Using a Class of Linear Inequalities." In Proceedings of the 15th IFAC Symposium Information Control Problems in Manufacturing, 2257-62.

[6] Gribaudo, M., Horvath, A., Bobbio, A., Tronci, E., Ciancamerla, E., and Minichino, M. 2003. "Fluid Petri Nets and Hybrid Model-Checking: A Comparative Case Study." Reliability Engineering and System Safety 81 (3): 239-57.
[7] Núñez, A., Pineda Cruz, J., and Sánchez, E. E. 2016. "Continuous Timed Petri Net Model of Brayton Cycle for a Gas Turbine.” In Proceedings of the 2016 International Conference on Control, Decision and Information Technologies (CoDIT).

[8] Valera, A. 2014. "Tecnologias Emergentes en Turbinas de Gas." Congresoy Exposicion en Turbomaquinaria.

[9] Thirunavukarasu, E. 2013. "Modeling and Simulation Study of a Dynamic Gas Turbine System in a Virtual Test Bed Environment." Master's thesis, University of South Carolina.

[10] Tovany, J. L., Ross-Leon, R., Ruiz-Leon, J., Ramirez-Treviño, A., and Begovich, O. 2013. "Greenhouse Modeling Using Continuous Timed Petri Nets." Mathematical Problems in Engineering.

[11] Alla, H., and David, R. 1998. "Continuous and Hybrid Petri Nets." Journal of Circuits, Systems and Computers.

[12] Recalde, L., Teruel, E., and Silva, M. 2005. “Autonomous Continuous p/t Systems." Application and Theory of Petri Nets.

[13] Wonham, W. 2013. "Supervisory Control of Discrete-Event Systems." Systems Control Group, ECE Dept, University of Toronto.

[14] Rodriguez, E. E. 2016. "Modal Model of Gas Turbine by Using Measurements of Vibration and Acoustics." Sub-mitted to ASME Turbo Expo.

[15] Julvez, J., and Mahulea, C. 2012 "SimHPN: A MATLAB Tool-Box for Hybrid Petri Nets." User Manual.

[16] Verma, M., and Kumar, A. 2014. "A Novel General Approach to Evaluating the Reliability of Gas Turbine System." Engineering Applications of Artificial Intelligence 28: 13-21. 